\title{
A potential therapeutic target miR-26b in tongue squamous cell carcinoma
}

\author{
Abu Ahmed ${ }^{1 *}$, Saied Mirshahidi ${ }^{2 *}$, Shohali Shammi ${ }^{1}$, Montry Suprono ${ }^{1}$ and Yiming Li $^{1}$ \\ ${ }^{1}$ Center for Dental Research, Loma Linda University School of Dentistry, A1019 Chan Shun Pavilion, 11175 Campus Street, Loma Linda, CA, USA \\ ${ }^{2}$ Department of Medicine \& Basic Sciences, Loma Linda University Cancer Center Biospecimen Laboratory, Loma Linda, CA, USA
}

\begin{abstract}
Objective: To determine microRNA expression in patients with Tongue squamous cell carcinoma (TSCC) and elucidate miR-26b significance in TSCC cell line. Study Design: Biochemical analysis of pathologic archive specimens.

Setting: Affiliated university laboratories.

Subjects and methods: The study included 18 patients with TSCC and TSCC tumor tissues and matching normal tissues were harvested from patients during tumor resection at the Loma Linda University Medical Center. MicroRNA (miRNA) expression analysis and its gain of function analysis were performed to understand miRNAs that were critical to the TSCC disease.

Result: miR-26b expression significantly decreased in TSCC tissues, when compared with normal tissues $(\mathrm{P}<0.0001)$. Likewise, reduced expression of miR-26b was observed in CAL27 cells (TSCC cell line) when compared with control OKF6/TERT-2 cell (B. P < 0.001). Overexpression of miR-26b significantly changes cellular 47 function like cell migration, cell proliferation, etc. tested on TSCC cell line, Cal27. It is even increasing the cisplatin activity on tongue squamous cell apoptosis when overexpressed.
\end{abstract}

Conclusion. Our study suggests that miR-26b plays a significant role in TSCC and might be a potential therapeutic target to regulate TSCC. Further investigation with in vivo studies and multiple TSCC cell lines are needed.

\section{Introduction}

Oral cancer is one of widely prevalent cancer types emerging as a growing problem in various regions of the world. As a global health problem, there are an estimated 657,000 new cases each year, with more than 330,000 deaths [1]. Approximately 53,000 people will be diagnosed with oral or oropharyngeal cancer this year in the US [2]. It will cause over 9,750 deaths, killing roughly 1 person per hour [2].

Tongue squamous cell carcinoma (TSCC) is the most common types of oral carcinoma and is characterized by a high risk of local recurrence and distant metastasis [3]. Although tobacco and alcohol drinking are responsible for the majority of new oral cancer cases, their prognostic role at the time of cancer presentation remains unclear [4]. Currently, surgical comprehensive therapy is the main treatment of TSCC, while surgery combined with radio- and/or chemotherapy is employed for advanced TSCC patients [5]. However, due to the side effects of chemotherapy and the distant metastasis, the prognosis is poor. The 5-year survival rate of patients with TSCC is around $67 \%$ [6]. However, recent research points out that there is an increased incidence of TSCC in the younger age group without any traditional risk factors making TSCC a distinct biological entity in this group [7]. Therefore, better understanding of the disease mechanisms will lead to discovery of early diagnosis, prognostic biomarkers, and development of more effective therapeutic strategies.

MicroRNAs (miRNAs) are single-strand RNAs comprising approximately 21-23 nucleotides, endogenously synthesized, noncoding RNAs, which are responsible for post-transcriptional regulation of mRNA expression [8,9]. There is now increasing evidence suggesting that a class of small non-coding RNAs, microRNAs (miRNAs), expressed in different tissues and cell types are involved in several physiological and pathological 80 conditions. MiRNA deregulation participates in pathogenesis of various cancers [10], including TSCC [11]. MiRNA expression profiling studies in TSCC patients have demonstrated that the expressions of a large number of miRNAs have been changed $[12,13]$. MiRNA contributes to a several cancer-related events, such as proliferation, migration, invasion and apoptosis [14], and abnormal expression of miRNAs 85 promotes drug resistance in cancers [15].

The miR-26b is classified as a member of the miR-26 family (miR$26 \mathrm{a} / \mathrm{b}$ ) and plays an important role in carcinogenesis and tumor progression by acting as a tumor-suppressor gene in multiple cancers [16-18]. MiR-26b is closely associated with the apoptosis and metastasis

${ }^{*}$ Correspondence to: Abu Ahmed, Center for Dental Research, Loma Linda University School of Dentistry, A1019 Chan Shun Pavilion, 11175 Campus Street, Loma Linda, CA, USA, Tel: (909) 651-5082, E-mail: aahmed@llu.edu

Saied Mirshahidi, Department of Medicine \& Basic Sciences, Loma Linda University Cancer Center Biospecimen Laboratory, Loma Linda, CA, USA, Tel: (909) 651-5082, E-mail: smirshahidi@llu.edu

Key words: MicroRNA, miR-26b, TSCC, Cal27, therapeutic target

Received: January 06, 2021; Accepted: January 21, 2021; Published: January 25, 2021 
of cancer cells. Several studies showed downregulated expression of miR-90 26b in TSCC [19-21]. However, the functional effect of miR$26 \mathrm{~b}$ related to TSCC progression needs further investigation.

Therefore, in this study, we first examined the expression level of miR-26b in tumor specimens from 18 TSCC patients. We also investigated the effect of miR-26b on cell proliferation, migration/ invasion, colony formation and induction of apoptosis in a TSCC cell line, Cal27, as well as exploring underlying molecular mechanism.

\section{Materials and methods}

\section{Patients and clinical samples}

TSCC tumor tissues and matching normal tissues were harvested from patients during tumor resection at the Loma Linda University Medical Center. Informed consent was obtained from all 18 patients. The tissues were collected, flash frozen and stored at $102-180^{\circ} \mathrm{C}$ by Loma Linda University Cancer Center Biospecimen Laboratory. Human tissue collection protocol was reviewed and approved by the local institutional review board (IRB\# 58238). The patients did not undergo radiotherapy, chemotherapy or other 105 targeted therapy before surgery.

\section{Cell culture}

Human keratinocyte cells (OKF6/TERT-2) and human TSCC cells (Cal27) were obtained from Dr. Jim Rheinwald Lab (Harvard Medical School, USA) and ATCC (CRL-2095, ATCC, USA) respectively. OKF6/ TERT-2 cells were cultured in Keratinocyte Serum Free Media (SFM) with supplement (ThermoFisher Scientific, USA) and Cal27 cells were cultured in Dulbecco's Modifed Eagle Medium (DMEM, ATCC, USA) added with fetal bovine serum (FBS; 10\%, HyClone, USA) and Penicillin-Streptomycin (1\%, ThermoFisher Scientific, USA) at $37^{\circ} \mathrm{C}$ with $5 \% \mathrm{CO}_{2}$.

\section{Transient transfection (Plasmids and transfection)}

Mimic of miR-26b (miR-26b) and the microRNA mimic negative control (miR-NC) were purchased from Dharmacon, Inc. (Lafayette, USA). Cal27 cells were seeded into 6-well plates (about $2 \times 10^{6}$ cells/well) and transfected with aforementioned nucleotides when confluence reached $70-80 \%$ using Cell Line Nucleofector Kit $\mathrm{V}$ and Nucleofector $2 \mathrm{~b}$ Device (Lonza Walkersville, Inc., MD, USA) according to the manufacturer's 122 recommendations. The efficiencies of cell transfection through electroporation were evaluated with the pmaxGFP vector and under a fluorescent microscope via GFP presence. In brief, Cal27 cells $\left(\cong 10^{6}\right)$ were resuspended in $100 \mu \mathrm{L}$ of the buffer 'V' solution with supplement, and $200 \mathrm{nM}$ of the human miR26b mimic (Dharmacon, Inc., IL, USA) were added for electric pulse. For control cells, we miR-NC of $200 \mathrm{nM}$ transfect cells (Dharmacon, Inc., IL, USA). After transfection, cells were gently resuspended in 2 $\mathrm{mL}$ of pre-warmed culture medium with $10 \%$ FBS and incubated for overnight at $37^{\circ} \mathrm{C}$ and $5 \% \mathrm{CO}_{2}$. Transfected cells were confirmed by the expression level of miR-26b using RNA isolation followed by RTqPCR.

\section{RT-qPCR}

The total RNA was isolated from tissues or transfected cells with miRNeasy Mini Kit (QIAGEN, Hilden, Germany) following the manufacturer's instructions. The RNA ( $5 \mu \mathrm{l})$ was directly converted to cDNA with the QuantiMir ${ }^{\mathrm{rm}}$ RT System (SBI System Biosciences, Mountain View, CA). Differential expression of miRNAs was analyzed by RTqPCR where U6 served as an internal control. For gene expression analysis, a portion of total RNA was used to synthesize cDNA using ThermoScript RT-PCR System (Qiagen, Hilden, Germany). All qPCR analysis were performed using SYBR-green reagents and gene expression levels was normalized to the internal reference expression level, beta-actin. The relative expression levels were assessed using the $2-$ $\Delta \Delta \mathrm{Ct}$ method. Primers sequences used were listed as below: miR-26bforward (F), 5'-CCGGGACCCAGTTCAAGTAA-3', miR-26b-reverse, 5'- CCCCGAGCCAAGTAATGGAG-3'; U6-F， 5'-CTCGCTTCGGCAGCACA-3', U6-R, 5'-AACGCTTCACGAATTTGCGT-3'; bActin-F, 5'- CAGGCATTGCTGACAGGATG-3', bActin-R, 5'- TGCTGATCCACATCTGCTGG-3'.

\section{Cell proliferation assay}

The MTT (3-[4,5-dimethylthiazol-2-yl]-2,5 diphenyl tetrazolium bromide) assay was applied to evaluate the effects of the overexpression of miR-26b in proliferation of Cal27 cells. The in vitro Cell Proliferation KIT 1 (Roche, 11465007001, Mannheim, Germany) was used. Cal27 cells from control and overexpressed miR-26b were seeded at a density of $5 \times 10^{3}$ cells/well in 96-well plate. After overnight culture, $10 \mu \mathrm{l}$ of MTT $(5153 \mu \mathrm{g} / \mathrm{ml}$ ) was added to every single well in the plate and incubated for 4 hours at $37^{\circ} \mathrm{C}$. Then $100 \mu$ of solubilization buffer (SDS $10 \%$ in $0.01 \mathrm{~N} \mathrm{HCl}$ ) was added to the wells and incubated at $37^{\circ} \mathrm{C}$ overnight. After the incubation, the absorbance was measured at 570 $\mathrm{nm}$ in a microplate reader, iMark (Bio-Rad, USA).

\section{Assessment of Cal27 migration by Boyden chamber assay}

Boyden chamber assays were carried out as previously described [22-24]. Briefly, Cal27 cells were grown in medium containing 10\% FBS for 48 hours post infection with miR- 26b or miR-NC. Subsequently, the treated cells were trypsinized and seeded into Boyden chambers (PET track-etched, 8 - $\mu$ m pores, 24 -well format; Becton Dickinson) in serum-free DMEM medium. Chambers were then immersed in $10 \%$ FBS medium for 5 hours. The topside of the membranes was swabbed to remove cells, and then cells on the bottom surface of the membrane were fixed with $4 \%$ paraformaldehyde, stained with DAPI to visualize nuclei, and counted under fluorescence microscopy. Five identically located fields per membrane were averaged for quantification of migrated cell numbers.

\section{Colony forming assay}

Control and transfected Cal27 cells were seeded in fresh media (200 cells/well of 6-well plate) and colony counting was performed to determine the colony forming potential of the adherent cells. Colonies were stained with $0.01 \%$ crystal violet (Sigma-Aldrich, Saint Louis, MO, USA) on day 14. Colonies containing at least 50 cells were counted. Experiments were done in triplicate.

\section{Apoptosis analysis}

Cell death by apoptosis was analyzed using the Annexin- $\mathrm{V}$ and propidium iodide (PI) apoptosis detection kit (BD Biosciences, San Jose, CA, USA). Briefly, mimic of miR-26b (miR-26b) and the microRNA mimic negative control (miR-NC) Cal27 cells were harvested and washed (both floating and attached cells). The cells were then resuspended in binding buffer at a density of $1-2 \times 10^{6}$ cells $/ \mathrm{ml}$. The $100 \mu \mathrm{l}$ single-cell suspension $\left(1-2 \times 10^{5}\right.$ cells $)$ was incubated with $5 \mu \mathrm{l}$ Annexin $\mathrm{V}$-fluorescein isothiocyanate for 20 minutes at room temperature and then $5 \mu$ l of PI was added at room temperature. Finally, the mixture was diluted with $500 \mu$ l binding buffer and analyzed with flow cytometry (MACSQuant, Miltenyi Biotec, Auburn, CA, USA). Using the FlowJo software (TreeStar, Ashland, Oregon, USA) the 
apoptotic rate was calculated as Annexin V+ cells. This experiment was repeated 3 times.

\section{Western blot analysis}

Western blotting was performed in accordance with a standard procedure using a polyclonal antibody that specifically recognized caspase 3 and Poly (ADP-ribose) polymerase-1 (PARP) (1:1000, Cell Signaling Technology, Danvers, MA, USA). Protein from the Cal27 cells were isolated from cell lysates using a radioimmunoprecipitation assay (Sigma) buffer supplemented with protease and phosphatase inhibitors. Following sonication and centrifugation, protein concentrations were quantified with the BCA assay. The extracted protein $(25 \mu \mathrm{g})$ was separated by $10 \%$ SDS-PAGE and transferred onto a polyvinylidene difluoride membrane (Millipore, Temecula, CA, USA). The membrane was blocked with $5 \%$ nonfat milk and then incubated with the caspase 3 antibody (1:1000, Cell Signaling Technology) in TBS buffer at $4^{\circ} \mathrm{C}$ overnight. The loading control was a GAPDH antibody (1:1000, Cell Signaling Technology, Danvers, MA, USA). After incubation with the secondary antibody with HRP goat anti-rabbit IgG (1:3000, EarthOx, Millbrae, CA, USA). Images were acquired by ImageQuant LAS 4000 Imaging Station (GE) and band densities were quantified using the ImageQuant TL software (GE). The data was normalized to corresponding values of GAPDH densitometry.

\section{Statistical analysis}

The data was analyzed with ANOVA using Prism 5.01 software (GraphPad Software, San Diego, CA, USA). A t-test was used for comparisons between two groups and ANOVA with Bonferroni was used for comparisons between more than two groups. A threshold value of $\mathrm{p}<0.05$ was considered statistically significant.

\section{Results}

\section{miR-26b was downregulated in TSCC tissues and cell lines}

The expression level miR-26b in 18 pairs of TSCC tissues (tumor tissue) and adjacent normal tissues (control) were initially detected using RT-qPCR. We found that miR-26b expression significantly decreased in TSCC tissues, when compared with normal tissues (Figure $1 \mathrm{~A}, \mathrm{P}<0.0001)$. Moreover, we also examined the expression of miR26b in TSCC cell line, Cal27 and in control cell line (OKF6/TERT-2). As shown in Figure 1B reduced expression of miR-26b was observed in CAL27 cells when compared with OKF6/TERT-2 cell (Figure 1B, P $<0.001)$.

\section{MiR-26b inhibits proliferation in TSCC cell line}

We found that the expression of miR-26b was highly reduced in TSCC tumor tissues and Cal27 cells. Cal27 cell line was selected for subsequent in vitro functional studies. First, expression of miR-26b in Cal27 cells transfected with miR-26b mimic or the microRNA mimic negative control (miR-NC) was measured by RT-qPCR (Figure 2A). RT-228 qPCR results revealed that the expression level of miR-26b was increased $>20$ fold in Cal27 cells transfected with miR-26b mimics (Figure 2A). In addition, the MTT assay revealed that Cal27 cells transfected with miR-26b mimics exhibited lower proliferative activity when compared with cells transfected with miR-NC (Figure 2B). In addition, clonogenic survival assay revealed that the colony formation ability was significantly hampered in Cal27 cells in response to upregulation of miR-26b expression compared $11 \mathrm{C}-\mathrm{D})$. These results indicate that miR-26b exerts a tumor suppressive function in TSCC cells. To the controls-(Figure 2C and Figure 2D). These results indicate thatmiR-26b exerts a tumor suppressive function in TSCC cells.

\section{Overexpressed miR-26b repress TSCC cell motility}

To examine whether overexpression of miR-26b in Cal27 cells can lead to decreases ininvasive potential, we next analyzed migration and invasion by Boyden chamber assay and wound healing assays. Overexpression of miR-26b in Cal27 cells significant lyattenuated cell motility when compared with the cells transfected with miR-NC (Figure 3A). Similar results were also observed in wound healing assay (Figure 3B).

\section{Manipulating miR-26b expression inducesapoptosis in Cal27 cells}

To investigate whether miR-26b is involved in regulating apoptosis, transfected Cal27cellswith miR-26b mimic (upregulation) or the microRNA mimic negative control (miR-NC) were harvested after 24 hours and stained with FITC-conjugated Annexin-V and PI and examined for apoptosis by flow cytometry. Quantitative analysis showed that in the presence of the miR-26b mimic (upregulation), total apoptotic $($ Annexin +$)$ cells increased significantly $\left({ }^{* * *} \mathrm{p}<0.001\right)$.
A

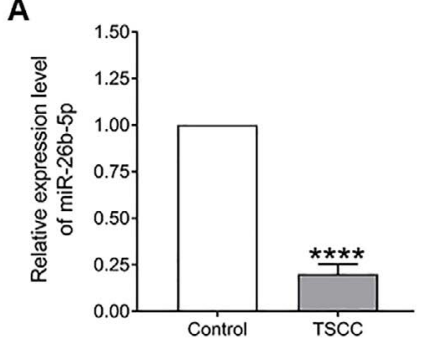

B

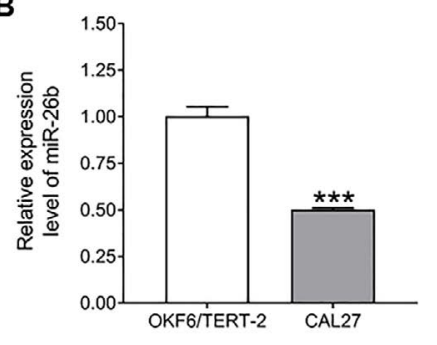

Figure 1. Relative expression of miR-26b in TSCC tumor tissues and cell lines. A) Decreased miR-26b expressions were observed in TSCC tumor tissues compared to normal matching tongue control $(\mathrm{n}=18)$. B) Decreased miR-26b-5p expressions were observed in TSCC cell line Cal27 compared to oral epithelial origin cell line OKF6/TERT-2 $(n=3)$. Relative expression of miR-26b-5p normalized to human U6 snRNA expression. Data are expressed as means \pm SEM as fold change from the normal matching tissue/control cell (OKF6/TERT-2), and statistical analysis using unpaired $t$ test was done with Prism software (Graphpad). (****P<0.0001, $\left.{ }^{* * * P} P<0.001\right)$.
A

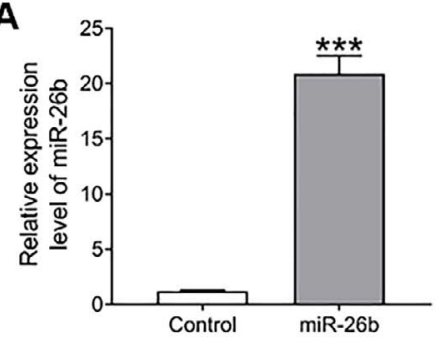

C

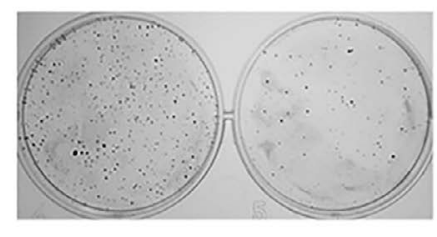

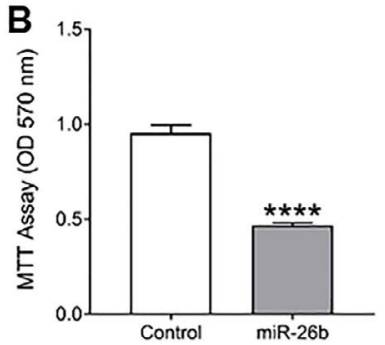

D

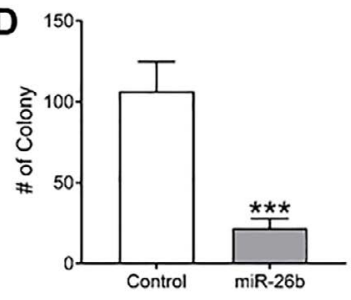

Figure 2. A) Verification efficiency of up-regulated miR-26b expression in Cal27 cells by RT-qPCR. B, After overexpression of miR-26b in Cal27 proliferation. C, Clonogenic cell survival assay. D, Number of colonies counting in clonogenic assay. $(* * * * P<0.0001$, $* * * P<0.001)$ 
A

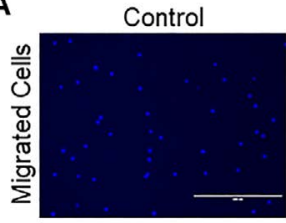

B

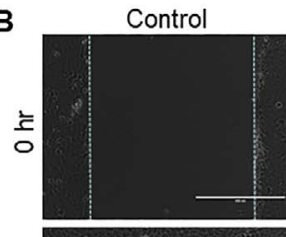

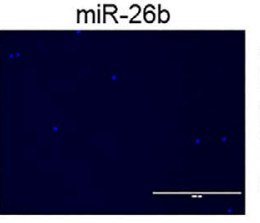
miR-26b

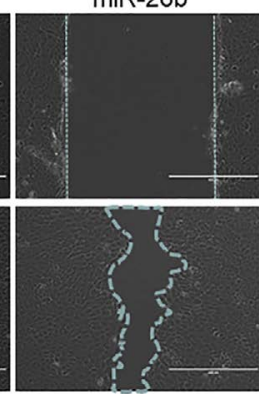

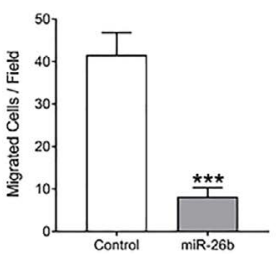

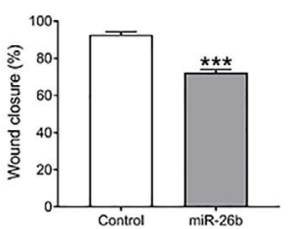

Figure 3. miR-26b inhibits Cal27 cells migration and invasion. A) Using Boyden chamber assay, cell migration was detected in Cal27 cells transfected with miR-26b mimic and miR-NC (negative control), respectively at $200 \times$ magnification. The migrated cells per field of the three groups is shown at the right. B) Wound healing assay in $\mathrm{Ca} 27$ cells where the ability of wound healing was decreased in over-expressed miR$26 \mathrm{~b}$ cells compared to negative control cells. The relative percentage of the wound closure of the three groups is shown at the right. Data are shown as mean $\pm \mathrm{SEM}$; $* * * \mathrm{P}<0.001$. Each experiment was repeated twice, and representative data from one such experiment are shown.

Flow cytometry was used to determine cell apoptosis following the manipulation of miR-26b expression in Cal27 (Figure 4) cells. In the presence of the miR-26b mimic(upregulation), the percentage of apoptotic cells increased by 2 folds $\left({ }^{* * *} \mathrm{p}<0.001\right)$, In addition, the miR-26b mimic and the miR-NCCal27 cells were exposed to $10 \mu \mathrm{M}$ cisplatin (Sigma) for 48 hours. As shown in Figure 4, upregulation of miR-26b significantly increased the cisplatin-induced apoptosis $(\mathrm{p}<0.05)$. These results suggest that miR-26b might be involved in regulating cell apoptosis [12].

\section{miR-26b expression link to apoptotic pathway}

To further elucidate the mechanisms of over-expressed miR-26binduced apoptosis in Cal27 cells, we evaluated the changes in expression of proteins involved in apoptosis such as PARP and caspase- 3 by Western blot analysis (Figure 4). We observed 260 decrease in the levels of caspase-3 and PARP (116 kD) expression in miR-26b over expressed Cal27 cells, which suggested that inhibitory effects might be correlated to cell apoptosis.

\section{Epidermal growth factor receptor (EGFR) was downregulated in TSCC tissues and cell lines}

The expression level EGFR in 18 pairs of TSCC tissues (tumor tissue) and adjacent normal tissues (control) were initially detected using RT-qPCR. We found that EGFR expression was significantly increased in TSCC tissues, when compared with normal tissues (Figure $6 \mathrm{~A}, \mathrm{P}<0.05)$. Moreover, we also examined the expression of EGFR in TSCC cell line, Cal27 and in control cell line (OKF6/TERT-2). As shown in Figure 6B induced expression of miR-26b was observed in CAL27 cells when compared with OKF6/TERT-2 cell (Figure 6B, $\mathrm{P}<$ 0.0001) (Table 1).

\section{Discussion}

MiRNAs may function as either oncogenes or tumor suppressors under certain conditions that have been shown to affect the hallmarks of cancer, including sustaining proliferative signalling, evading growth suppressors, activating invasion and metastasis, resisting cell death, and inducing angiogenesis [25]. In our study, we validated that miR-278 $26 \mathrm{~b}$ was downregulated in TSCC tumor tissues from 18 cases of TSCC patients and 13 TSCC cell line, which was consistent with previous reports $[19,21]$. MiR-26b was revealed to be downregulated in many multiple carcinoma [16-18,26,27], indicating the tumour suppressive property of this miRNA.

In order to assess the functional impact of miR-26b in cancer cell, miR-26b was overexpressed in TSCC cell line, Cal27, using gene electroporation method to study the role of miR-26b in the cellular behaviours, including cell proliferation, migration, invasion, and apoptosis. As the continual unregulated proliferation of cancer cells is

Table 1. Clinicopathological features of the TSCC patients* (T: Tumor; N: Nodes; M: Metastasis; NA: Not Available).

\begin{tabular}{|c|c|c|c|c|c|c|c|}
\hline No. & Age & Sex & $\begin{array}{c}\text { Tumor size } \\
(\mathbf{c m})\end{array}$ & $\mathbf{T}^{*}$ & $\mathbf{N}^{*}$ & $\mathbf{M}^{*}$ & Differentiation \\
\hline 1 & 60 & Male & 5.0 & 4 & 0 & 0 & Moderate \\
\hline 2 & 70 & Male & NA & 1 & 0 & 0 & Moderate \\
\hline 3 & 55 & Female & 1.5 & 1 & 0 & 0 & Moderate \\
\hline 4 & 68 & Male & 4.2 & $4 \mathrm{a}$ & $2 \mathrm{c}$ & 0 & Poor \\
\hline 5 & 51 & Male & 6.7 & 3 & $2 \mathrm{~b}$ & 0 & Moderate \\
\hline 6 & 72 & Female & 3.9 & 2 & 0 & 0 & Moderate \\
\hline 7 & 52 & Male & 2.3 & 2 & 1 & 0 & Moderate \\
\hline 8 & 49 & Male & 4.0 & 4 & 1 & 0 & Well-Moderate \\
\hline 9 & 70 & Male & NA & 2 & 0 & 0 & Moderate \\
\hline 10 & 81 & Female & 4.4 & 3 & 0 & 0 & Well \\
\hline 11 & 64 & Female & 1.7 & 1 & 1 & 0 & Poor \\
\hline 12 & 54 & Male & 5.1 & 3 & $2 \mathrm{c}$ & 0 & Moderate-Poor \\
\hline 13 & 54 & Female & 1.8 & 1 & 0 & 0 & Moderate \\
\hline 14 & 66 & Male & NA & 4 & $2 \mathrm{c}$ & 0 & Moderate-Poor \\
\hline 15 & 58 & Male & NA & $4 \mathrm{a}$ & $2 \mathrm{c}$ & 0 & Moderate \\
\hline 16 & 53 & Female & 4.3 & 3 & 0 & 0 & Moderate \\
\hline 17 & 90 & Male & 3.3 & 2 & 0 & 0 & Moderate \\
\hline 18 & 60 & Male & 5.5 & 3 & $2 \mathrm{~b}$ & 0 & Moderate \\
\hline & & & & & & & \\
\hline
\end{tabular}
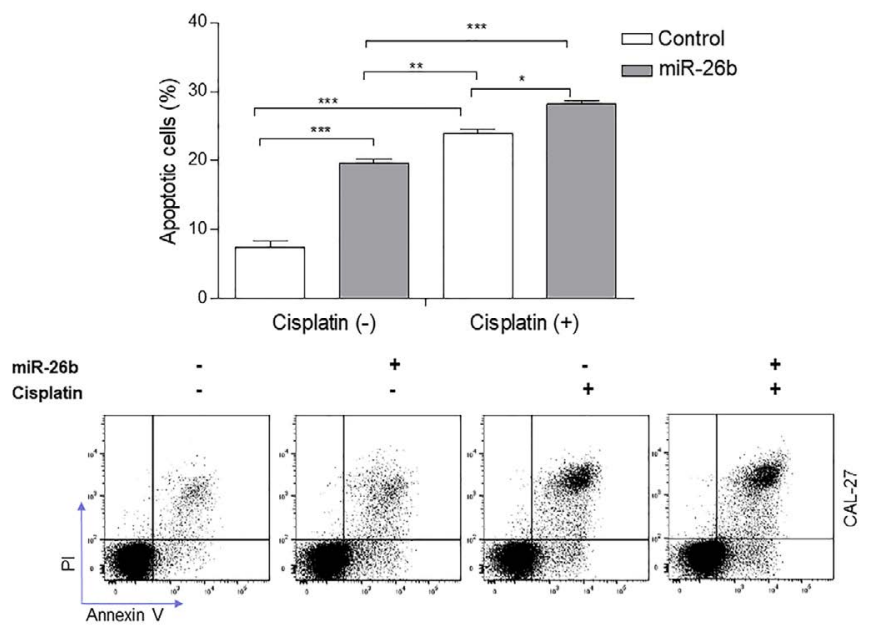

Figure 4. The assessment of apoptosis in Cal27 cells treated with cisplatin or mimics of miR-26b by flow cytometry (annexin V/PI). Cal27 cells were treated with mimics of miR-26b in the absence/presence of $10 \mu \mathrm{M}$ cisplatin for $48 \mathrm{~h}$ analysis (down). The bar chart (up) shows the ratios of apoptosis cell numbers between the groups treated with the negative control mimic, miR-26b mimic, negative control mimic+cisplatin or miR-26b mimic + cisplatin. Data represent the mean of three independent experiments \pm SEM with $n=3$. $* P<0.05 ; * * P<0.01 ; * * * P<0.001$. 
A

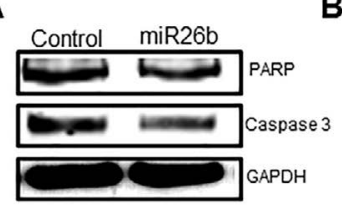

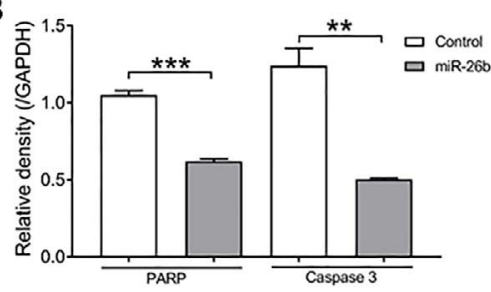

Figure 5. A, Expression of PARP and caspase 3 by Western blotting. B, Relative density of A. Expression of PARP and caspase 3 in the overexpressed miR-26b group was lower than in the control group. There were significant differences between the two groups. ${ }^{* *} P<0.01$ and $* * * P<0.001$. (one-way ANOVA).
A

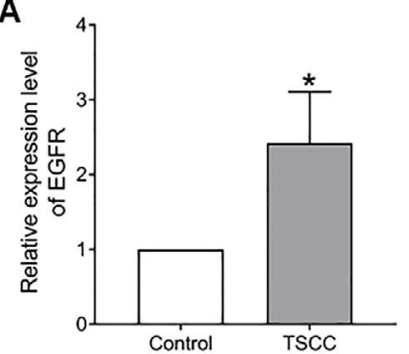

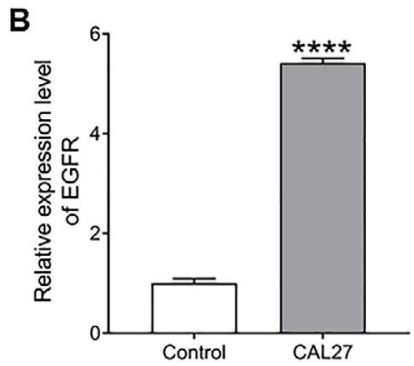

Figure 6. A) Relative expression of EGFR in TSCC tumor tissues compared to normal matching tissues $(n=17)$. B) Relative expression of EGFR in TSCC cell line, Cal27 compared to normal cell line, OKF6/TERT-2 of oral epithelial origin $(n=6)$. Data are expressed as means $\pm \mathrm{SEM}$, and statistical analysis using unpaired $t$ test was done with Prism software (Graphpad). $\left({ }^{*} P<0.05, * * * P<0.0001\right)$.

the fundamental abnormality resulting in the development of cancer [28], in the current study, we observed, overexpression of miR-26b that significantly inhibited the proliferation of Cal27 cell in vitro. Similarly, we found that the upregulation of miR-26b-mediated suppression of cell migration and invasion, indicating the functional role of miR-26b in TSCC. Overexpression of miR-26b in Cal27 shows the significant apoptosis compared to control cells. Caspase- 3 is involved in both the intrinsic and extrinsic apoptotic pathways. During apoptosis PARP protein is cleaved by caspases (most likely caspase-3). The Western blot data showed that in miR-26b over-expressed Cal27 cells the level of uncleaved caspase- 3 decreased, which implies that the level of active caspase- 3 increased. In addition, the level of uncleaved PARP was also decreased, which demonstrates that PARP cleaved occurred.

Cisplatin is the most commonly used chemotherapy drug in TSCC treatment $[29,30]$. However, chemotherapy resistance occurs during the course of treatment, resulting in more aggressive tumors, metastasis, and a poor prognosis [31]. We demonstrated that miR$26 \mathrm{~b}$ overexpression in Cal27 cells the significantly increased cisplatininduced apoptosis. Therefore, miR-26b could potentially act as a novel therapeutic molecule to combat chemotherapy resistance.

We also found that epidermal growth factor receptor (EGFR) expression was significantly reduced in TSCC tissues, when compared with normal tissues (Figure 6A, P $<306$ 0.05). Similarly, we found the expression of EGFR was decreased in TSCC cell line, Cal27, when compared with OKF6/TERT-2 cells (Figure 6B, $\mathrm{P}<0.001$ ). As EGFR is one of the most potent oncogenes that are commonly altered in cancers [32], there might be a correlation between miR-26b and EGFR that causes TSCC. In fact, De Robertis et al. [33], showed that EGFR gene, linked to a possible control by miR-26b, could be proposed as novel colorectal cancer (CRC) prognostic biomarkers. Further studies are needed to prove whether miR-26b can be utilized as a novel diagnostic and prognostic biomarker in TSCC patients.
The limitations of our study include the fact that this is an in vitro study. The behaviour of MicroRNAs overexpression in the in vivo setting cannot be assumed based on in vitro 316 studies. Further in vivo studies should be performed to evaluate the tumor suppressive activity of the overexpressed miR-26b in rodent tongue cancer model.

\section{Conclusion}

Within limitations of the present study, we demonstrated that miR-26b is relatively downregulated in TSCC tissues and miR-26b has regulatory role in the modulation of a variety of pathophysiological processes. These results indicate that miR-26b may serve as a tumorsuppressor gene involved in human TSCC and might function as therapeutic target for these patients and might be combined with other current conventional treatments.

\section{Funding source}

This work was supported by the Center for Dental Research, LLUSD internal fund.

\section{References}

1. WHO (2021) Oral Cancer. Available from: https://www.who.int/cancer/prevention/ diagnosis-screening/oral-cancer/en/

2. The Oral Cancer Foundation (2021) Oral Cancer Facts. Available from: https:// oralcancerfoundation.org/facts/

3. Chang B, He W, Ouyang H, Peng J, Shen L, et al. (2018) A Prognostic Nomogram Incorporating Depth of Tumor Invasion to Predict Long-term Overall Survival for Tongue Squamous Cell Carcinoma With R0 Resection. $J$ Cancer 9: 2107-2115. [Crossref]

4. Beynon RA, Lang S, Schimansky S, Penfold CM, Waylen Aet al. (2018) Tobacco smoking and alcohol drinking at diagnosis of head and neck cancer and all-cause mortality: Results from head and neck 5000, a prospective observational cohort of people with head and neck cancer. Int J Cancer 143: 1114-1127. [Crossref]

5. Lai H, Xu G, Meng H, Zhu H (2019) Association of SP1 rs1353058818 and STAT3 rs 1053004 gene polymorphisms with human tongue squamous cell carcinoma. Biosci Rep 39: BSR20190955.

6. National Cancer Institute (2021) SEER Cancer Statistics Review 1975-2017. Available from: https://seer.cancer.gov/csr/1975_2017/results_single/sect_20_table.11.pdf

7. Mohideen K, Krithika C, Jeddy N, Bharathi R, Thayumanavan B, et al. (2019) Metaanalysis on risk factors of squamous cell carcinoma of the tongue in young adults. $J$ Oral Maxillofac Pathol 23: 450-457. [Crossref]

8. Lou S, Sun T, Li H, Hu Z (2018) Mechanisms of microRNA-mediated gene regulation in unicellular model alga Chlamydomonas reinhardtii. Biotechnol Biofuels 11: 244 [Crossref]

9. Si W, Shen J, Zheng H, Fan W (2019) The role and mechanisms of action of microRNAs in cancer drug resistance. Clin Epigen 11: 25.

10. Rupaimoole R, Calin GA, Lopez-Berestein G, Sood AK (2016) miRNA Deregulation in Cancer Cells and the Tumor Microenvironment. Cancer Discov 6: 235-246. [Crossref]

11. Jia LF, Wei SB, Gong K, Gan YH, Yu GY (2013) Prognostic implications of micoRNA miR-195 expression in human tongue squamous cell carcinoma. PLoS One 8: e56634. [Crossref]

12. Schneider A, Victoria B, Lopez YN, Suchorska W, Barczak W, et al. (2018) Tissue and serum microRNA profile of oral squamous cell carcinoma patients. Sci Rep 8: 675. [Crossref]

13. Zhou XL, Wu JH, Wang XJ, Guo FJ (2015) Integrated microRNA-mRNA analysis revealing the potential roles of microRNAs in tongue squamous cell cancer. $\mathrm{Mol} \mathrm{Med}$ Rep 12: 885-894. [Crossref]

14. Volinia S, Calin GA, Liu CG, Ambs S, Cimmino A, et al. (2006) A microRNA expression signature of human solid tumors defines cancer gene targets. Proc Natl Acad Sci U S A 103: 2257-2261. [Crossref]

15. Tormo E, Ballester S, Adam-Artigues A, Burgués O, Alonso E, et al. (2019) The miRNA-449 family mediates doxorubicin resistance in triple-negative breast cancer by regulating cell cycle factors. Sci Rep 9: 5316. [Crossref] 
16. Liu XX, Li XJ, Zhang B, Liang YJ, Zhou CX, et al. (2011) MicroRNA-26b is underexpressed in human breast cancer and induces cellapoptosis by targeting SLC7A11. FEBS Lett 585: 1363-1367. [Crossref]

17. Zhang C, Tong J, Huang G (2018) Nicotinamide phosphoribosyl transferase (Nampt) is a target of microRNA-26b in colorectal cancer cells. PloS One 8: e69963.

18. Wu N, Zhao X, Liu M, Liu H, Yao W, et al. (2011) Role of microRNA-26b in glioma development and its mediated regulation on EphA2. PloS One 6: e16264. [Crossref]

19. Cao J, Guo T, Dong Q, Zhang J, Li Y (2015) miR-26b is downregulated in human tongue squamous cell carcinoma and regulates cell proliferation and metastasis through a COX-2-dependent mechanism. Oncol Rep 33: 974-980. [Crossref]

20. Jia LF, Wei SB, Gan YH, Guo Y, Gong K, et al. (2014) Expression, regulation and roles of miR-26a and MEG3 in tongue squamous cell carcinoma. Int J Cancer 135: 2282-2293. [Crossref]

21. Wei Z, Chang K, Fan C, Zhang Y (2020) MiR-26a/miR-26b represses tongue squamous cell carcinoma progression by targeting PAK1. Cancer Cell Int 20: 1-14.

22. Wang X, Hu G, Zhou J (2010) Repression of versican expression by microRNA-143. J Biol Chem 285: 23241-23250.

23. Wang X, Hu G, Gao X, Wang Y, Zhang W, et al. (2012) The induction of yes-associated protein expression after arterial injury is crucial for smooth muscle phenotypic modulation and neointima formation. Arterioscler Thromb Vasc Biol 32: 2662-2669. [Crossref]

24. Xu F, Ahmed ASI, Kang X, Hu G, Liu F, et al. (2015) MicroRNA-15b/16 attenuates vascular neointima formation by promoting the contractile phenotype of vascular smooth muscle through targeting YAP. Arterioscler Thromb Vasc Biol 35: 2145-2152. [Crossref]
25. Peng Y, Croce CM (2016) The role of MicroRNAs in human cancer. Signal Transduct Target Ther 1: 1-9. [Crossref]

26. Schultz NA, Dehlendorff C, Jensen BV, Bjerregaard JK, Nielsen KR, et al. (2013) MicroRNA biomarkers in whole blood for detection of pancreatic cancer. JAMA 311: 392-404. [Crossref]

27. Ji J, Shi J, Budhu A, Yu Z, Forgues M, et al. (2009) MicroRNA expression, survival, and response to interferon in liver cancer. $N$ Engl J Med 361: 1437-1447. [Crossref]

28. Cooper GM, Hausman RE (2000) The development and causes of cancer. The cell: A molecular approach. pp: 2.

29. Zhong LP, Zhang CP, Ren GX, Guo W, William Jr WN, et al. (2013) Randomized phase III trial of induction chemotherapy with docetaxel, cisplatin, and fluorouracil followed by surgery versus up-front surgery in locally advanced resectable oral squamous cell carcinoma. J Clin Oncol 31: 744. [Crossref]

30. Gibson MK, Li Y, Murphy B, Hussain MHA, DeConti RC, et al. (2005) Randomized phase III evaluation of cisplatin plus fluorouracil versus cisplatin plus paclitaxel in advanced head and neck cancer (E1395): an intergroup trial of the Eastern Cooperative Oncology Group. J Clin Oncol 23: 3562-3567. [Crossref]

31. Wu G, Wilson G, George J, Liddle C, Hebbard L, et al. (2017) Overcoming treatmen resistance in cancer: Current understanding and tactics. Cancer Lett 387: 69-76. [Crossref]

32. Thomas R, Weihua Z (2019) Rethink of EGFR in Cancer With Its Kinase Independent Function on Board. Front Oncol 9: 800.

33. De Robertis M, Loiacono L, Fusilli C, Poeta ML, Mazza T, et al. (2017) Dysregulation of EGFR pathway in EphA2 cell subpopulation significantly associates with poor prognosis in colorectal cancer. Clin Cancer Res 23: 159-170. [Crossref]

Copyright: $\odot 2021$ Ahmed A. This is an open-access article distributed under the terms of the Creative Commons Attribution License, which permits unrestricted use, distribution, and reproduction in any medium, provided the original author and source are credited. 